Ann. Biol. anim. Bioch. Biophys., I973, 13 (4), 627-64I.

\title{
L'UROLITHIASE OVINE EFFETS D'UN PHOSPHONATE DE SODIUM ET DU CHLORURE D'AMMONIUM
}

\author{
J.-P. BARLET, M. THÉRIEZ* et G. MOLÉNAT* \\ avec la collaboration technique de Mme BELLANGer et J.-M. MANSART \\ Station de Physiopathologie de la Nutrition, \\ * Station de Recherches sur l'Élevage, des Ruminants, \\ Centre de Recherches de Clermont-Ferrand, I. N.R. A., \\ Theix 63110 Beaumont
}

\section{RÉSUMÉ}

Nous avons essayé de mettre en évidence les modifications du métabolisme phosphocalcique survenant chez l'agneau atteint d'urolithiase et de prévenir l'apparition de ces troubles par l'utilisation d'un phosphonate de sodium (EHDP) ou de chlorure d'ammonium, tout en observant l'influence de l'EHDP ou de $\mathrm{NH}_{4} \mathrm{Cl}$ sur les performances zootechniques et le métabolisme phosphocalcique de jeunes agneaux mâles, de races diverses, recevant une ration à base de maîs ou de sorgho.

Au cours de deux expériences successives nous avons utilisé respectivement 4 lots de I 2 agneaux (lots $A, B, C$ et $D$ ) et 3 lots de $I 5$ agneaux ( $E, F$ et $G$ ). Les lots $A, E, F$ et $G$ recevaient un concentré à base de maiss, les lots $\mathrm{B}, \mathrm{C}$ et $\mathrm{D}$ un concentré à base de sorgho. Les animaux des lots $C$ et $G$ recevaient en outre quotidiennement, par voie orale, ro mg d'EHDP par $\mathrm{kg}$ de poids vif et le concentré distribué aux animaux des lots $D$ et $F$ était additionné de I p. Ioo de $\mathrm{NH}_{4} \mathrm{Cl}$.

Dans I'expérience I, 6 animaux du lot A et 5 animaux du lot B ont été atteints d'urolithiase, tandis qu'aucun cas n'a pu être décelé parmi les animaux des lots C et D. L'apparition de l'urolithiase semblait toujours précédée par une élévation de la phosphaturie. Les variations des paramètres sanguins étudiés ne paraissaient pas susceptibles de fournir des indications sûres permettant de prévoir l'apparition des calculs urinaires. L'EHDP diminuait significativement la phosphaturie et le taux rénal de phosphore. $\mathrm{Le} \mathrm{NH}_{4} \mathrm{Cl}$ augmentait la calciurie, la rétention calcique et le volume d'urine excrété quotidiennement, tout en abaissant le $\mathrm{pH}$ urinaire.

Dans l'expérience II, nous n'avons observé aucun cas d'urolithiase et aucun calcul urinaire n'a pu être décelé à l'abattage, même chez les témoins (lot $\mathrm{E}$ ). Cependant, la croissance journalière des animaux du lot $\mathrm{E}$ était significativement inférieure à celle des animaux des lots $\mathrm{F}$ et $\mathrm{G}$. De plus, chez les témoins, il existait une corrélation négative hautement significative entre la croissance moyenne journalière et la teneur rénale en calcium. Ceci semblait indiquer, chez les témoins, la présence d'urolithiase à un niveau subclinique, laquelle était inhibée par l'EHDP ou le $\mathrm{NH}_{4} \mathrm{Cl}$. 


\section{INTRODUCTION}

L'urolithiase ovine, qui sévit principalement chez les animaux soumis à un régime alimentaire intensif, est caractérisée par la présence d'un sédiment minéral cristallisé dont les éléments, de taille variable, sont constitués essentiellement par des phosphates de magnésium ou de calcium. Ce sédiment est localisé dans les tubes rénaux, les bassinets, la vessie et l'urèthre. Chez les mâles, la présence de ce sédiment dans l'urèthre peut conduire à l'obstruction de celui-ci, ce qui provoque finalement la distension et la rupture de la vessie, entraînant ainsi la mort de 1'animal (JEANBLAIN et al., I968). L'étiologie de ces troubles est inconnue. Il semble cependant que dans certains cas une teneur élevée en phosphore de la ration, avec un rapport $\mathrm{Ca} / \mathrm{P}$ voisin de $\mathrm{I}$, favorise l'apparition de l'urolithiase. Selon certains travaux, le sorgho aurait un effet calculogénique plus important que celui du maîs ou des autres céréales (UDALI, et CHEN CHOW, I969). Les essais de prophylaxie de l'urolithiase basés sur l'addition d'un sel de sodium, calcium, potassium ou ammonium à la ration ont donné des résultats variables selon les auteurs (FIELD, I969; UDALI, et CHEN CHow, I969). Il semble cependant que le chlorure d'ammonium soit le plus efficace (CROokshank, I966, I970).

Chez 1'Homme, les pyrophosphates ont été utilisés dans la prévention des calculs urinaires (FLEISCH, BISAZ et CARE, I964). En effet, utilisés in vitro, à très faible concentration $\left(\mathrm{ro}^{-6} \mathrm{M}\right)$ ils inhibent la précipitation du phosphate calcique (Fleisch et NeUman, I96I). Chez le Rat, 1'administration parentérale de pyrophosphate permet de prévenir la calcification de l'aorte induite expérimentalement par administration de vitamine $\mathrm{D}_{3}$ (ScmrbLER, RUSSEL et FLEISCH, I968). Les pyrophosphates pourraient donc avoir un rôle préventif à l'égard de l'urolithiase ovine. Malheureusement, le pyrophosphate n'est pas résorbé lorsqu'il est administré oralement et il est détruit très rapidement lorsqu'il est administré par voie parentérale (JUNG et al., I970). Les diphosphonates, qui présentent une liaison $\mathrm{P}-\mathrm{C}-\mathbf{P}$ au lieu de la liaison $\mathrm{P}-\mathrm{O}-\mathrm{O}$ du pyrophosphate, sont résistants à l'hydrolyse chimique et enzymatique (FLEISCH et BonJOUR, I97I). Deux phosphonates, 1'éthane-I-hydroxyr,I-diphosphonate (EHDP) et le dichlorométhylène-diphosphonate préviennent les calcifications aortiques et rénales induites par la vitamine $\mathrm{D}$ chez le Rat (FLEISCH et al., I970). De plus, chez le Rat, l'EHDP diminue la croissance des calculs urinaires induits par différents procédés (FRASER et al., I97I). Il semblait donc intéressant d'étudier l'influence du chlorure d'ammonium et du phosphonate sur le métabolisme phosphocalcique de l'Agneau, tout en observant leur rôle préventif éventuel à l'encontre des calculs urinaires.

\section{MATÉRIEL, ET MÉTHODES}

Au cours de deux expériences successives, nous avons offert à des agneaux mâles sevrés, à l'engraissement, des rations riches en céréales.

Pour mettre en évidence le rôle préventif des traitements expérimentaux, nous avons établi des régimes destinés à favoriser l'apparition des cas de lithiase (utilisation du sorgho lors de la première expérience et rapport phosphocalcique des aliments variant de I à $\mathbf{I}, 5$ ). 
Les deux traitements préventifs suivants ont été comparés, d'une part l'apport de chlorure d'ammonium ( 1 p. xoo de la matière sèche) et d'autre part l'administration de phosphonate (ro $\mathrm{mg} / \mathrm{kg}$ de poids vif) aux animaux.

$\mathrm{Au}$ cours de la première expérience, nous avons déterminé le bilan minéral des agneaux recevant les différents régimes et, au cours des deux essais, nous avons comparé l'évolution des teneurs en $\mathrm{P}, \mathrm{Ca}, \mathrm{Mg}$ et $\mathrm{Cl}$ du sang et celle du $\mathrm{pH}$ urinaire.

Nous avons également mesuré la vitesse de croissance des animaux et leur consommation de foin et d'aliment concentré pour mettre en évidence le rôle éventuel des traitements.

\section{Animaux}

L'apparition des cas de lithiase pouvant être plus ou moins fréquente selon le type génétique des agneaux, nous avons utilisé des animaux de plusieurs races, sevrés précocement au cours de leur $7^{\mathrm{e}}$ semaine et pesant $\mathrm{I}_{2}$ à $I_{4} \mathrm{~kg}$ en début d'expérience.

Au cours du premier essai, nous avons réparti 48 agneaux en quatre lots expérimentaux constitués chacun de 4 Limousins, 4 Romanov $\times$ Limousins, 2 Berrichons $\times$ (Romanov-Limousins) et 2 Charmois.

Au cours du second essai les 45 agneaux utilisés ont été répartis en 3 lots de 5 soit 9 Charmois et 6 croisés Hampshive $\times$ (Romanov-Limousins).

\section{Aliments}

La formule et la composition chimique des aliments sont reportés sur le tableau $I$.

Au cours du I er essai, nous avons comparé le maîs et le sorgho dans deux lots témoins. Bien que celui-ci soit moins fréquemment utilisé en France que le maîs, nous l'avons cependant retenu

TABLEAU I

Formule et composition minérale des aliments distribués aux agneaux

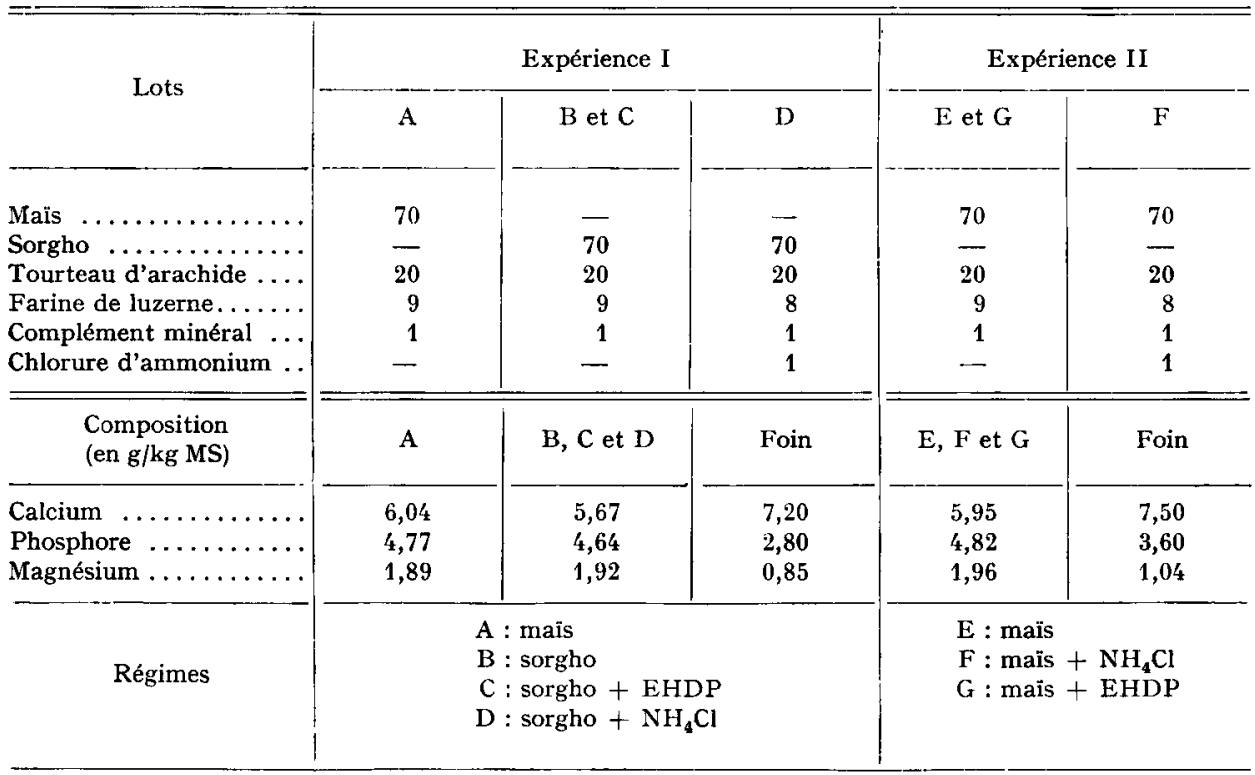

Composition du complément minéral (p. 100)

Carbonate de calcium : 60,79; Chlorure de sodium : 23,00; Sulfate de magnésium : 15,00; Sulfate de cuivre : 0,20; Sulfate de zinc : 0,60 ; Sulfate de cobalt : 0,01 ; Sulfate de manganèse : 0,40 . 
car les cas de lithiase sont plus fréquents chez les agneaux qui sont engraissés avec des aliments qui en contiennent. C'est également sur des animaux consommant les aliments à base de sorgho que nous avons essayé les deux traitements préventifs expérimentaux.

Cet essai a été répété sur des animaux recevant des aliments à base de maîs.

Dans les 2 essais nous avons offert aux agneaux du foin de prairie naturelle de qualité moyenne.

\section{Conduite de l'expérience}

Tous les agneaux élevés en lots, sur litière de paille et disposant d'eau en permanence, recevaient l'aliment concentré à volonté. Celui-ci était apporté tous les jours, les refus étant ramassés et pesés deux fois par semaine. La quantié de foin offerte était limitée à zo p. roo de la quantité de concentré lors du premier essai, à $50 \mathrm{p}$. Ioo lors du second.

Chaque jour les agneaux des lots $C$ et $G$ recevaient Io $\mathrm{mg} / \mathrm{kg}$ de poids vif de phosphonate dissous dans $20 \mathrm{ml}$ d'eau et administré à la seringue par voie orale.

Les agneaux étaient pesés chaque semaine à jour et heure fixes et abattus, soit lorsque leur poids atteignait $32 \mathrm{~kg}$ pour les Charmois et $35 \mathrm{~kg}$ pour les animaux des autres races ou croisements, soit lorsqu'ils présentaient des symptômes de lithiase urinaire.

Lors du premier essai, au moment de chaque pesée on prélevait $20 \mathrm{ml}$ de sang par ponction de la veine jugulaire. Une quantié analogue d'urine était également recueillie. Le pH de l'urine était déterminé immédiatement au pH-mètre, et le sang centrifugé. Le plasma recueilli était conservé à - I $5^{\circ} \mathrm{C}$ jusqu'au moment de l'analyse. Lors du second essai nous avons prélevé du sang en début et fin d'expérience seulement.

Lors de l'abattage des animaux nous avons examiné le système urinaire de tous les agneaux et prélevé le rein gauche et un fragment médian du métacarpien droit pour dosage des éléments minéraux.

\section{Méthodes d'analyse et détermination du bilan minéral des agneaux}

Quatre agneaux Limousins de chacun des régimes ont été prélevés dans les lots et placés en cage à bilan ro ou 25 jours après le début de la première expérience. Les animaux recevaient alors la même alimentation que celle dont ils disposaient en lot et, après 5 jours d'adaptation aux cages, nous avons déterminé leur bilan minéral. Au cours des ro jours de mesure, les quantités d'aliments ingérées, le volume d'urine et le poids de fèces excrétées étaient mesurés chaque matin.

Sur les différents échantillons recueillis, nous avons dosé le calcium et le magnésium par spectrophotométrie d'absorption atomique, le phosphore minéral par colorimétrie du complexe phospho-vanado molybdique, le chlore par potentiométrie.

Ces différentes mesures ont été effectuées après dégraissage par l'acétone puis minéralisation des échantillons osseux et rénaux. Les échantillons d'aliments, de fèces et d'urine ont également été minéralisés alors que les dosages ont été faits sur le plasma sans traitement préalable.

\section{RÉSULTATS}

\section{A. - Fréquence des cas de lithiase}

Le nombre de cas de lithiase apparus lors de l'expérience I dans les lots $\mathrm{A}$ (mais : 6 cas) et B (sorgho : 5 cas) n'est pas significativement différent. Par contre, la fréquence des cas aigus de lithiase (animaux abattus ou morts en cours d'expérience) ou le nombre d'agneaux présentant des calculs dans la vessie lors de l'abattage est significativement plus élevé lors de l'essai I dans les lots A (maîs) et $\mathrm{B}$ (sorgho) que dans les lots $\mathrm{C}$ (sorgho $+\mathrm{EHDP}$ ) et $\mathrm{D}$ (sorgho $+\mathrm{ClNH}_{4}$ ) dans lesquels aucun agneau n'a présenté de troubles $\left(\left(\chi^{2}\right.\right.$ respectivement égal à 4 et $5, P<0,05$ dans les deux cas).

Lors du $2^{\text {e }}$ essai nous n'avons observé aucun symptôme clinique et aucun animal ne présentait de calculs dans la vessie lors de l'abattage. 


\section{B. - Quantités d'aliments ingérées et croissance des agneaux}

Lors du I er essai, II agneaux ayant été atteints de lithiase dans les lots A et B, nous les avons abattus dès l'apparition des symptômes. Cependant, il n'a pas été possible de préciser la durée de la période de formation, période au cours de laquelle les animaux ont pu avoir un comportement différent. C'est pourquoi il n'est pas possible de comparer valablement les quantités d'aliments ingérées par les animaux en fonction du régime.

De même, les cas de lithiase étant apparus sur des animaux de types génétiques différents, les croissances des agneaux indemnes (tabl. 2) ne peuvent être comparées d'un lot à l'autre car ceux-ci ne sont plus homogènes au point de vue race.

Les agneaux atteints de lithiase ont eu des croissance très variables au cours des I5 derniers jours avant l'abattage, $3 I 7 \mathrm{~g} / \mathrm{j}$ pour les animaux du lot maïs, $86 \mathrm{~g} / \mathrm{j}$ pour ceux du lot sorgho avec des écarts individuels importants de $-80 \mathrm{~g} / \mathrm{j}$ à $+425 \mathrm{~g} / \mathrm{j}$. Il n'est donc pas possible d'utiliser la variation de poids d'un animal entre 2 pesées pour déterminer le moment d'abattage des animaux avant l'apparition des symptômes cliniques.

TABLEAU 2

Quantités d'aliments ingérées et vitesse de croissance des agneaux

\begin{tabular}{|c|c|c|c|c|c|}
\hline & \multirow[b]{2}{*}{ Lots } & \multicolumn{4}{|c|}{ Aliments } \\
\hline & & $\begin{array}{c}\text { Aliment concentré } \\
(\mathrm{kg} / \mathrm{agneau} / \mathrm{j})\end{array}$ & $\begin{array}{c}\text { Foin } \\
(\mathrm{kg} / \mathrm{agneau} / \mathrm{j})\end{array}$ & $\begin{array}{l}\text { Gain quotidien } \\
\text { (g/agneau/j) }\end{array}$ & $\begin{array}{c}\text { Indice } \\
\text { de consommation } \\
(\mathrm{kg} \text { de } \mathrm{MS} / \mathrm{agneau} / \mathrm{j})\end{array}$ \\
\hline & & \multicolumn{4}{|c|}{ Expérience I } \\
\hline $\mathbf{A}$ & Maïs témoin & 0,86 & 0,23 & 208 & \\
\hline B & Sorgho témoin..... & 0,88 & 0,25 & 234 & \\
\hline C & Sorgho + EHDP . & $.0,94$ & 0,25 & 242 & \\
\hline \multirow[t]{2}{*}{ I) } & Sorgho $+\mathrm{NH}_{4} \mathrm{Cl} \ldots$ & 0,98 & 0,31 & 231 & \\
\hline & & \multicolumn{4}{|c|}{ Expérience II } \\
\hline $\mathbf{E}$ & Mais $\ldots \ldots \ldots \ldots$ & 1,069 & 0,159 & $185 \pm 67$ & 6,32 \\
\hline I & Maïs $+\mathrm{NH}_{4} \mathrm{Cl} \ldots$ & 1,129 & 0,147 & $247 \pm 62^{*}$ & 5,02 \\
\hline (i & Maïs + EHDP ... & 1,093 & 0,148 & $230 \pm 67^{*}$ & 5,43 \\
\hline
\end{tabular}

Signification statistique : * $\mathrm{P}<0,05$.

Comparaison par rapport aux valeurs obtenues chez les animaux du lot $\mathrm{E}$.

Au contraire, aucun animal n'ayant présenté de symptôme de lithiase au cours du second essai, il est possible de comparer les consommations et les croissances des animaux des différents lots. L'addition de chlorure d'ammonium ou de EHDP n'a 
pas entraîné de réduction des quantités d'aliment ingérée. Ces deux traitements ont, au contraire, permis d'améliorer significativement la croissance des animaux $(\mathrm{P}<0,05)$ et par là de réduire leur indice de consommation. Chez ro animaux du lot $\mathrm{E}$, choisis au hasard, il existe une corrélation négative hautement significative $(P<0,0 I)$ entre la teneur du rein en calcium et la croissance moyenne journalière (fig. I).

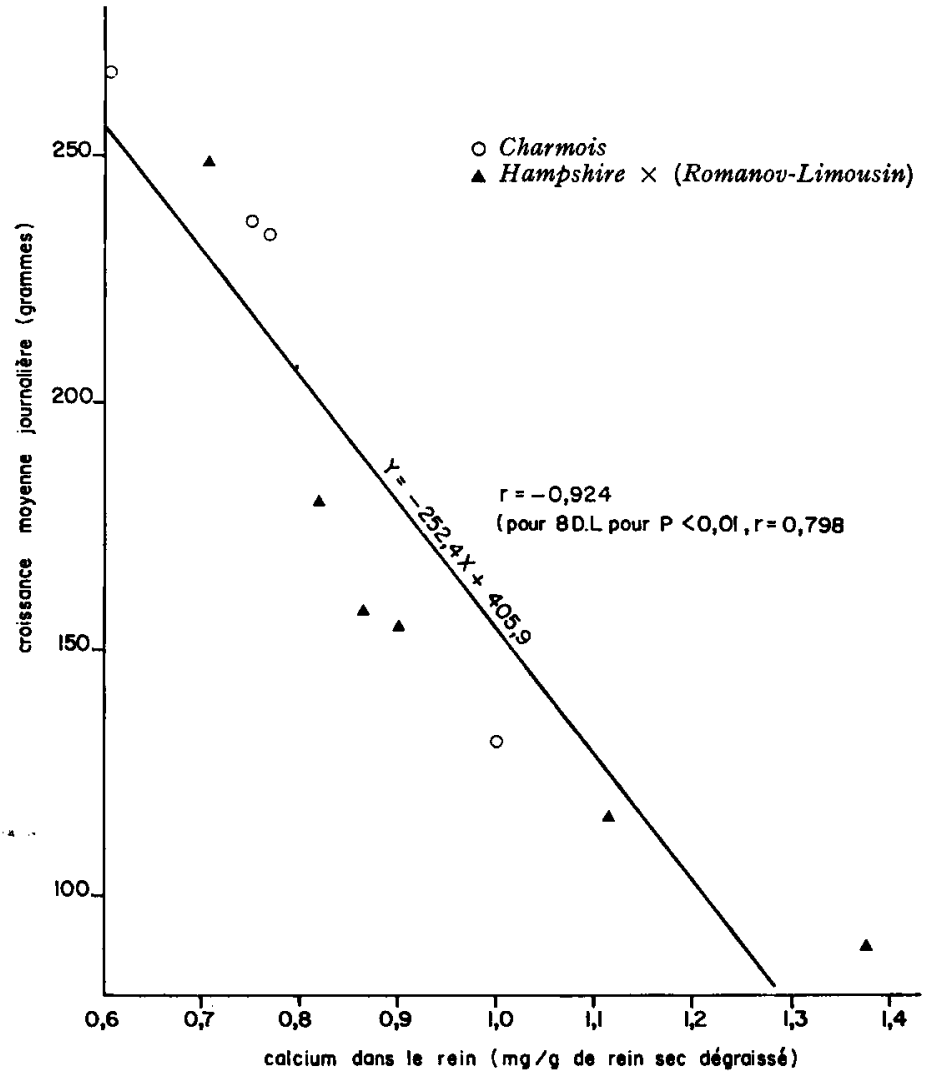

FIG. I. - Corrélation entre le taux renal de calcium et la croissance moyenne journalière chez dix agneaux alimentês au maïs (lot $E$ ).

\section{C. - Infuence de la lithiase sur les paramètres minéraux sanguins et urinaires}

Chez les 7 agneaux qui ont présenté, pendant les mesures de bilan, des symptômes cliniques de l'urolithiase et chez lesquels des calculs urinaires ont été décelés à l'abattage, l'excrétion urinaire quotidienne de phosphore est significativement plus élevée $(\mathrm{P}<0, \mathrm{oI})$ que chez les témoins (tabl. 3). Par contre, il n'existe pas de différence significative en ce qui concerne l'excrétion urinaire du calcium ou du magnésium, celle-ci étant très variable chez les animaux atteints de lithiase (tabl. 3). Chez ces derniers, le volume d'urine excrétée en $24 \mathrm{~h}$ est légèrement supérieur $(964 \pm$ Ior $\mathrm{ml})$ à celui des animaux normaux $(867 \pm 204 \mathrm{ml})$, mais la différence n'est pas significative. 
TABLEAU 3. - Influence des différents régimes ou de l'urolithiase sur les paramètres du métabolisme minéral

(expérience I) $\left(\bar{x} \pm \frac{\sigma}{\sqrt{\mathrm{N}}}\right)$

\begin{tabular}{|c|c|c|c|c|c|}
\hline Lots & Éléments & $\begin{array}{c}\text { Quantité ingérée } \\
(\mathrm{g} / \mathrm{j})\end{array}$ & $\begin{array}{c}\text { Excrétion fécale } \\
(\mathrm{g} / \mathrm{j})\end{array}$ & $\begin{array}{c}\text { Excrétion urinaire } \\
(\mathrm{mg} / \mathrm{j})\end{array}$ & $\begin{array}{l}\text { Rétention } \\
(\mathrm{g} / \mathrm{j})\end{array}$ \\
\hline $\begin{array}{c}A \text { (maïs) } \\
(4)\end{array}$ & $\begin{array}{c}\mathrm{Ca} \\
\mathrm{P} \\
\mathrm{Mg}\end{array}$ & $\begin{array}{l}3,89 \pm 0,46 \\
2,98 \pm 0,76 \\
1,95 \pm 0,25\end{array}$ & $\begin{array}{l}2,89 \pm 0,67 \\
1,64 \pm 0,79 \\
0,87 \pm 0,38\end{array}$ & $\begin{array}{c}26,73 \pm 9,46 \\
54,38 \pm 11,64 \\
270,40 \pm 100,17\end{array}$ & $\begin{array}{l}0,99 \pm 0,64 \\
1,13 \pm 0,19 \\
0,81 \pm 0,29\end{array}$ \\
\hline $\begin{array}{c}\text { B (sorgho) } \\
(4)\end{array}$ & $\begin{array}{c}\mathrm{Ca} \\
\mathbf{P} \\
\mathrm{Mg}\end{array}$ & $\begin{array}{l}4,16 \pm 0,37 \\
3,04 \pm 0,19 \\
2,04 \pm 0,11\end{array}$ & $\begin{array}{l}2,75 \pm 0,49 \\
1,87 \pm 0,64 \\
1,00 \pm 0,17\end{array}$ & $\begin{array}{c}23,49 \pm 1,30 \\
48,29 \pm 8,73 \\
246,38 \pm 83,37\end{array}$ & $\begin{array}{l}1,37 \pm 0,39 \\
1,07 \pm 0,64 \\
0,74 \pm 0,12\end{array}$ \\
\hline $\begin{array}{c}\mathrm{C} \text { (sorgho }+ \text { EHDP) } \\
(4)\end{array}$ & $\begin{array}{c}\mathrm{Ca} \\
\mathrm{P} \\
\mathrm{Mg}\end{array}$ & $\begin{array}{l}4,29 \pm 0,41 \\
3,27 \pm 0,38 \\
2,13 \pm 0,19\end{array}$ & $\begin{array}{l}1,88 \pm 0,67 \\
2,04 \pm 0,79 \\
1,08 \pm 0,29\end{array}$ & $\begin{array}{r}32,54 \pm 4,43^{*} \\
7,48 \pm 4,59^{* *} \\
389,19 \pm 158,67\end{array}$ & $\begin{array}{l}2,36 \pm 0,51^{*} \\
1,08 \pm 0,54 \\
0 ; 70 \pm 0,39\end{array}$ \\
\hline $\mathrm{D}$ (sorgho $+\mathrm{NH}_{4} \mathrm{Cl}$ ) & $\begin{array}{c}\mathrm{Ca} \\
\mathrm{P} \\
\mathrm{Mg}\end{array}$ & $\begin{array}{l}3,96 \pm 0,38 \\
3,64 \pm 0,71 \\
1,98 \pm 0,27\end{array}$ & $\begin{array}{l}1,64 \pm 0,30^{*} \\
1,90 \pm 0,71 \\
0,89 \pm 0,28\end{array}$ & $\begin{array}{r}54,27 \pm 8,36^{* *} \\
12,37 \pm 9,56^{* *} \\
351,69 \pm 78,24\end{array}$ & $\begin{array}{l}2,46 \pm 0,61^{* *} \\
1,71 \pm 0,59 \\
0,79 \pm 0,25\end{array}$ \\
\hline $\begin{array}{l}\text { Agneaux présentant } \\
\text { des calculs urinaires } \\
\text { (7) }\end{array}$ & $\begin{array}{c}\mathrm{Ca} \\
\mathrm{P} \\
\mathrm{Mg}\end{array}$ & $\begin{array}{l}4,68 \pm 1,04 \\
3,29 \pm 0,91 \\
2,04 \pm 0,49\end{array}$ & $\begin{array}{l}3,47 \pm 0,88 \\
2,20 \pm 0,46 \\
0,98 \pm 0,33\end{array}$ & $\begin{array}{c}19,44 \pm 3,71 \\
228,40 \pm 164,49^{* *} \\
589,64 \pm 268,40\end{array}$ & $\begin{array}{l}1,59 \pm 0,44 \\
1,04 \pm 0,71 \\
0,85 \pm 0,33\end{array}$ \\
\hline
\end{tabular}

Signification statistique : ${ }^{*} \mathrm{P}<0,05 ; \quad{ }^{* *} \mathrm{P}<0,01$ (test $t$ de Student) (comparaison par rapport aux animaux du lot B).

TABLEAU 4. - Composition minérale des reins

(mg/g de rein sec dégraissé, $\left(\bar{x} \pm \frac{\sigma}{\sqrt{\mathrm{N}}}\right)$

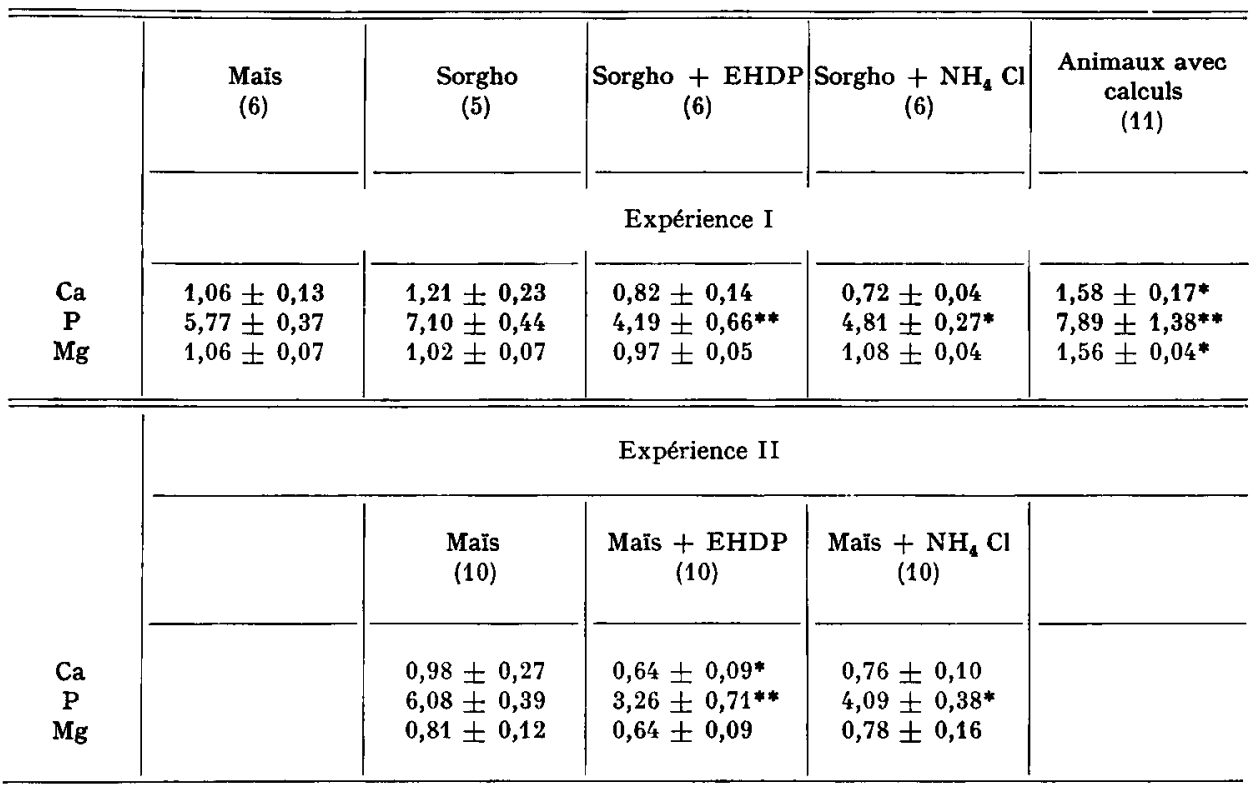

Signification statistique : * $\mathrm{P}<0,05 \quad * * \mathrm{P}<0,01$ (test $t$ de Student); comparaison par rapport aux animaux recevant le même régime, sans addition d'EHDP ou de $\mathrm{NH}_{4} \mathrm{Cl}$.

Les chiffres entre parenthèses indiquent le nombre d'échantillons utilisés. 
Chez ces 7 agneaux le taux rénal de calcium, magnésium et phosphore est significativement supérieur $(\mathrm{P}<\mathrm{O}, \mathrm{OI})$ à celui des témoins (tabl. 4), alors qu'il n'existe pas de différence significative en ce qui concerne la teneur des métacarpes en ces éléments (tabl. 5).

\section{TABLEAU 5}

Composition minérale des os $\left(\bar{x} \pm \frac{\sigma}{\sqrt{\mathrm{N}}}\right)$

(mg/g d'os sec dégraissé)

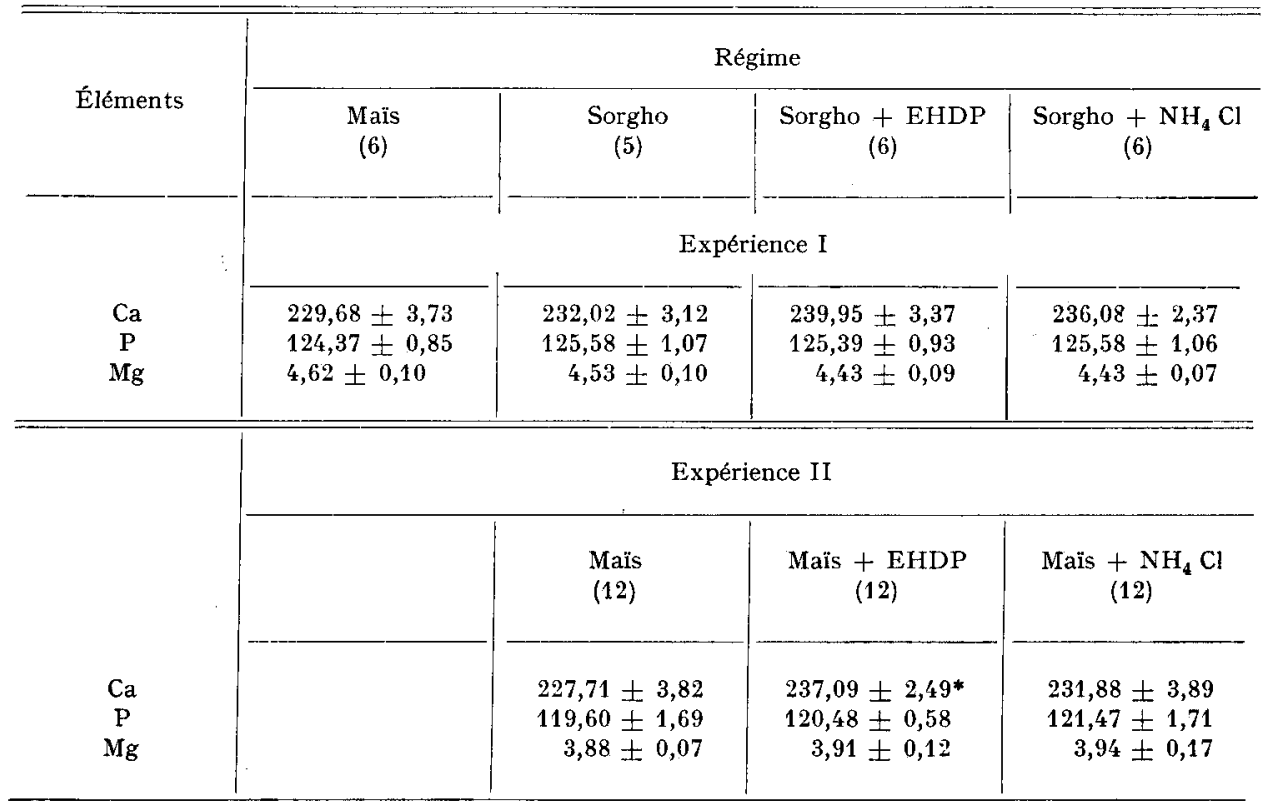

Signification statistique : ${ }^{*} \mathrm{P}<0,05$ (test $t$ de Student). Comparaison par rapport au "régime sorgho " (expérience I) ou au "régime maïs " (expérience II).

\section{D. - Influence de l'EHDP}

L'EHDP n'a pas d'influence significative sur le volume d'urine excrétée (824 $\pm 107 \mathrm{ml} / 24 \mathrm{~h}$ chez les animaux recevant l'EHDP, $808 \pm 99 \mathrm{ml} / 24 \mathrm{~h}$ chez les témoins). L'EHDP diminue significativement $(P<0,0 I)$ l'excrétion urinaire du phosphore, augmente significativement celle du calcium $(\mathrm{P}<0,05)$ et n'a pas d'effet significatif sur celle du magnésium (tab1. 3). Chez les animaux nourris au sorgho (lot C), l'administration quotidienne d'EHDP abaisse significativement la phosphatémie $(\mathrm{P}<0,05)$ et élève significativement la calcémie $(\mathrm{P}<0,0 \mathrm{I})$. Ces différences significatives apparaissent $28 \mathrm{j}$ après le début du traitement (tabl. 6).

A l'abattage, la teneur en calcium des os des agneaux ayant reçu l'EHDP n'est pas significativement différente de celle des témoins (tabl. 5). Par contre, 


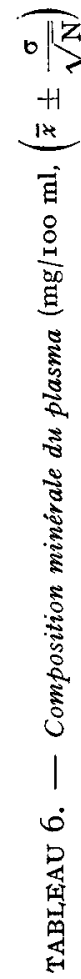

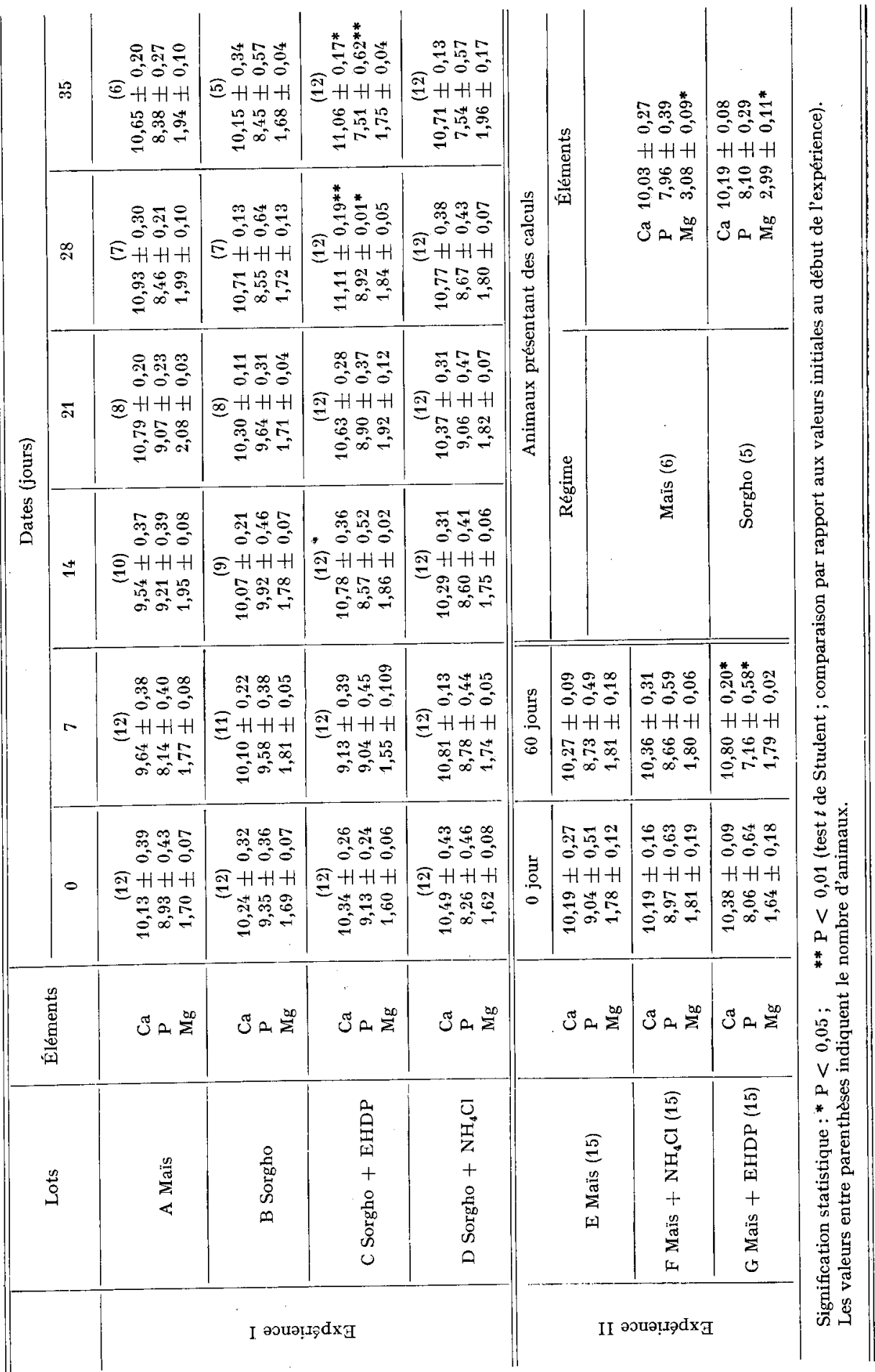


l'EHDP diminue significativement $(\mathrm{P}<0,05)$ la teneur du tein en calcium et en phosphore (tabl. 4).

Dans l'expérience II, la phosphatémie des animaux ayant reçu l'EHDP (lot $\mathrm{G})$ est significativement plus faible $(\mathrm{P}<\mathrm{o}, \mathrm{or})$ à l'abattage qu'au début de 1'expérience, alors que la calcémie est significativement augmentée $(\mathrm{P}<0,0 \mathrm{I})$. La teneur en calcium du métacarpien de ces animaux est significativement $(P<0,05)$ supérieure à celle des témoins (tabl. 5). Comme dans l'expérience I, la teneur du rein en calcium et en phosphore est significativement $(P<0,05)$ abaissée par rapport à celle des témoins (tabl. 4).

\section{E. - Influence $d u \mathrm{NH}_{1} \mathrm{Cl}$}

La quantité journalière d'urine émise par les agneaux recevant le $\mathrm{NH}_{4} \mathrm{Cl}$ ( $237 \pm \operatorname{I09} \mathrm{ml} / 24 \mathrm{~h}$ ) est significativement supérieure $(\mathrm{P}<0,05)$ à celle émise par les témoins $(867 \pm 204 \mathrm{ml} / 24 \mathrm{~h}$ ). Dès 7 jours après le début du traitement, le $\mathrm{pH}$ de l'urine émise par les animaux recevant le $\mathrm{NH}_{4} \mathrm{Cl}$ est significativement inférieur à sa valeur initiale (tabl. 7). Le $\mathrm{NH}_{4} \mathrm{Cl}$ augmente significativement la calciurie $(P<O, O I)$ et diminue significativement la phosphaturie $(P<0, o r)$, alors qu'il est sans effet significatif sur la magnésiurie (tab1. 3). Il diminue significativement

\section{TABLEAU 7}

Influence des divers régimes sur le pH de l'urine émise par les animaux

\begin{tabular}{|c|c|c|c|c|c|c|}
\hline \multirow{3}{*}{ Régime } & \multicolumn{6}{|c|}{ Date } \\
\hline & \multicolumn{6}{|c|}{ Expérience I } \\
\hline & 0 & 7 jours & 14 jours & 21 jours & 28 jours & 35 jours \\
\hline $\begin{array}{l}\text { Maïs } \ldots \ldots \ldots \ldots \ldots \\
\text { Sorgho } \ldots \ldots \ldots \ldots \\
\text { Sorgho }+ \text { EHDP } \\
\text { Sorgho }+\mathrm{NH}_{4} \mathrm{Cl}\end{array}$ & $\begin{array}{l}8,05(4) \\
7,90(6) \\
7,90(5) \\
7,95(6)\end{array}$ & $\begin{array}{l}7,95(5) \\
7,90(5) \\
7,90(4) \\
7,10(5)^{*}\end{array}$ & $\begin{array}{l}8,00(4) \\
8,05(4) \\
8,00(4) \\
7,00(6)^{*}\end{array}$ & $\begin{array}{l}8,00(4) \\
7,95(5) \\
7,95(5) \\
7,00(5)^{*}\end{array}$ & $\begin{array}{l}8,00(4) \\
8,00(5) \\
8,00(6) \\
6,95(6)^{*}\end{array}$ & $\begin{array}{l}7,95(4) \\
7,95(4) \\
7,95(4) \\
6,90(4)^{* *}\end{array}$ \\
\hline & \multicolumn{6}{|c|}{ Expérience $I I$} \\
\hline & 0 & \multicolumn{4}{|c|}{30 jours } & 50 jours \\
\hline $\begin{array}{l}\text { Mais } \ldots \ldots \ldots \ldots \\
\text { Mais }+ \text { EHDP } \ldots \\
\text { Maïs }+\mathrm{NH}_{4} \mathrm{Cl} \ldots\end{array}$ & $\begin{array}{l}7,95(6) \\
8,00(6) \\
8,00(6)\end{array}$ & & \multicolumn{2}{|c|}{$\begin{array}{l}8,00(6) \\
7,95(6) \\
6,85(6)^{*}\end{array}$} & & $\begin{array}{l}8,02(6) \\
8,00(6) \\
6,80(6)^{* *}\end{array}$ \\
\hline
\end{tabular}

Signification statistique : ${ }^{*} \mathrm{P}<0,05 ; * * \mathrm{P}<0,01$ (test $t$ de STUdent). Comparaison par rapport aux valeurs obtenues chez les mêmes animaux le jour du début de l'expérience. 
l'excrétion fécale du calcium $(\mathrm{P}<0,05)$ et augmente significativement la rétention calcique $(\mathrm{P}<0,05)$ tabl. 3). L'administration quotidienne de $\mathrm{NH}_{4} \mathrm{Cl}$ n'a aucun effet significatif sur la calcémie, la phosphatémie et la magnésie des animaux (tabl. 6). Il n'a pas non plus d'effet significatif sur la chlorémie.

\section{DISCUSSION}

\section{Étiologie de l'urolithiase}

Dans les jours qui précèdent l'apparition des symptômes cliniques de l'urolithiase, l'Agneau excrète une quantité de phosphore urinaire très supérieure à celle observée chez les animaux sains recevant un régime analogue : ceci confirme les résultats obtenus par différents auteurs chez le Bélier (PACKETT, LiNERBERGER et JaCrson, I968) ou chez l'Agneau (RobBins, KUnKEL et Crookshank, I965). Les causes de cette excrétion accrue de phosphore urinaire sont encore inconnues à l'heure actuelle. On peut cependant envisager que la calcitonie et (ou) la parathormone puissent intervenir puisque ces deux hormones, utilisées à dose physiologique, se sont révélées hyperphosphaturiantes chez l'Agneau recevant une ration à base de céréales (BARLET, I972 ; BARLET et CARE, I972). De plus, chez la Souris et chez le Chien, une néphrocalcinose peut être induite expérimentalement par injections répétées de parathormone (BERRY, I970); de même, chez le Singe, un hyperparathyroïdisme induit expérimentalement stimule fortement l'apparition de dépôts calciques au niveau du rein (DrACr et BOYCE, I972).

Il a également été signalé que la calcémie des agneaux atteints d'urolithiase était supérieure à celle des témoins indemnes (PACKETt et Hauschild, I964). Dans notre expérimentation nous n'avons pas trouvé de différences significatives entre la calcémie et la phosphatémie des animaux présentant des calculs et celles des témoins ; seule la magnésémie chez certains animaux atteints d'urolithiase (4 sur 7) était supérieure à celle des témoins.

Chez le Rat, l'apparition des calculs urinaires formés de phosphate d'ammonium peut être prévenue par supplémentation de la ration en magnésium (GouLDING et MaLthus, I969); chez l'Agneau une telle supplémentaton s'est révélée favorable (Bushman, EMERICK et EMBry, r965) ou sans effet significatif (ThÉRIEz, Moléénat et BARLET, données non publiées).

\section{Effets prophylactiques de l'EHDP}

Chez les agneaux nourris au sorgho, l'EHDP a eu un effet préventif à l'égard de l'urolithiase dans nos essais. Il semble que ce phosphonate soit capable d'agir à doses très faibles puisque les travaux effectués chez l'Agneau à l'aide du ${ }^{14} \mathrm{C}-\mathrm{E} H \mathrm{HP}$ ont montré que des animaux de $30 \mathrm{~kg}$, recevant per os, $300 \mathrm{mg}$ d'EHDP en absorbaient entre 2,5 et $3 \mathrm{mg}$. $\mathrm{La}$ fraction non absorbée est éliminée sous forme d'EHDP dans les fèces et $50 \mathrm{p}$. IOo environ de 1'EHDP ayant franchi la paroi intestinale sont excrétés dans l'urine (ROSENBLUM et BARLET, données non publiées). 
Le métabolisme de l'EHDP semble donc analogue chez le Ruminant et chez le Monogastrique (MrCHAEL, KING et WAKIN 1972). Chez le Rat, l'EHDP s'est révélé efficace dans la prévention des calculs urinaires formés de phosphate ou d'oxalate de calcium, mais sans effet net sur le développement des calculs constitués de phosphate de magnésium (FraSER et al., I97I) : cependant les conditions expérimentales étaient très différentes de celles que nous avons utilisées chez l'Agneau, puisque chez le Rat, l'apparition des calculs était induite par implantation de fragments de zinc dans la vessie. On peut penser que l'effet préventif que nous avons observé avec le phosphonate est lié à la diminution de l'excrétion urinaire du phosphore minéral, puisque l'EHDP n'a aucun effet significatif sur le volume out le $\mathrm{pH}$ urinaire.

Au niveau de l'os, à la dose utilisée, 1'EHDP a tendance à augmenter la teneur en calcium du métacarpien ; ce résultat est en accord avec la diminution de l'excrétion fécale du calcium, et l'effet parait d'autant plus intense que le traitement est plus prolongé (tabl. 5). Chez le Rat, l'effet de l'EHDP au niveau de l'anabolisme et du catabolisme osseux varie beaucoup selon la dose utilisée (KIng, Francis et MICHAEL, I97I ; FRASER et al., I972). La légère baisse de la calcémie et de la phosphatémie observée en fin d'expérimentation chez les deux lots d'agneaux ayant reçu l'EHDP pourrait d'ailleurs résulter d'une inhibition du catabolisme osseux. En effet, chez le Rat, l'EHDP administré à la dose de $7,5 \mathrm{mg} / \mathrm{kg} / \mathrm{j}$ pendant ${ }_{4}$ jours augmente significativement la teneur de l'os en minéraux (KING, Francis et MichaEL, I97I).

\section{Effet prophylactique du $\mathrm{NH}_{4} \mathrm{Cl}$}

Le $\mathrm{NH}_{4} \mathrm{Cl}$ a, comme l'EHDP, un effet préventif significatif à l'égard de l'urolithiase chez l'Agneau recevant du sorgho. Chez 1'Homme, le $\mathrm{NH}_{4} \mathrm{Cl}$ est connu. pour élever la calcémie et la magnésiurie (LAVAN, I969). Chez le Mouton castré de $\mathrm{I}$ an, l'administration orale de $\mathrm{NH}_{4} \mathrm{Cl}(0,2 \mathrm{~g} / \mathrm{kg} / \mathrm{j})$ pendant $70 \mathrm{j}$ augmente la calcémie et le taux d'absorption calcique intestinale, tout en abaissant le $\mathrm{pH}$ urinaire (BRATTHWAITE, I972), sans modifier la taille des compartiments liquidiens de l'organisme (MANSTON, I972). Nous avons retrouvé des résultats analogues chez nos animaux ayant reçu la ration à base de sorgho supplémenté en $\mathrm{NH}_{4} \mathrm{Cl}$. Chez le Rat, une élévation du $\mathrm{pH}$ urinaire associée à une diminution de la magnésiurie semble constituer un facteur important dans l'étiologie de la néphrocalcinose (GroRy et al., 1970). Chez cet animal, le $\mathrm{NH}_{4} \mathrm{Cl}$, en abaissant le $\mathrm{pH}$ de l'urine prévient efficacement. 1'apparition de tels troubles rénaux (Gour,DING et MALTHUS, I970). Il pourrait en être de même chez l'Agneau, chez lequel, en outre ce sel possède un effet diurétique et calciurique important (tabl. 3 et 7 ). Cependant, chez l'Homme, l'excrétion urinaire du calcium ne semble pas influencer beaucoup l'apparition des calculs urinaires. (Bulusu et al., I970). Chez le Mouton, la teneur de la ration en phosphore semble être un facteur étiologique de l'urolithiase plus important que le $\mathrm{pH}$ de l'urine. (HoAr, Emerick et Embry, I970). Cependant, le chlorure d'ammonium possède un rôle préventif indéniable dans certaines conditions expérimentales (CrookSHANK, I966, I970) : il semble que cela soit confirmé par notre expérimentation. Selon certains auteurs (UDALL et CHENCHOW, I965) l'action préventive du $\mathrm{NH}_{4} \mathrm{Cl}$ serait liée à la présence de l'ion chlore plutôt qu'à celle de l'ion ammonium, puisque le chlorure de sodium peut également être utilisé avec succès dans la prophylaxie de l'urolithiase. Néanmoins, la chlorémie des animaux présentant des calculs. 
urinaires (104,25 $\pm \mathrm{I,32}$ méqv/1) est analogue à celle des témoins normaux (I03,05 $\pm 0,78$ méqv/1).

On pourrait alors penser que le $\mathrm{NH}_{4} \mathrm{Cl}$ agit en augmentant la consommation d'eau par l'animal, provoquant ainsi une diurèse accrue (ELAM, HAM et SchNEIDER, 1957). En réalité la consommation quotidienne d'eau des 4 animaux recevant le $\mathrm{NH}_{4} \mathrm{Cl}$, mesurée pendant ro jours, était légèrement supérieure à celle des témoins $(2,4 \pm 0,201 / j$ contre $2,2 \pm 0,351 / j)$ mais la différence n'était pas significative. De même les 7 animaux chez lesquels des calculs urinaires ont été mis en évidence n'avaient pas une consommation journalière d'eau significativement différente de celle des témoins. L'effet diurétique du $\mathrm{NH}_{4} \mathrm{Cl}$ ne résulte donc sans doute pas uniquement d'une augmentation de la consommation d'eau par les animaux. D'ailleurs le volume journalier d'urine excrétée par des agneaux soumis à un régime calculigène est supérieur à celui des témoins recevant un régime normal (PACKETT et BỨTCHER, I963; PACKE'TT et al., rg68). De même, chez l'Homme, l'acétazolamide est connu comme pouvant induire l'apparition de dépôts de phosphate de calcium au niveau du rein, tout en augmentant le volume d'urine excrétée (PARFITT, I969).

\section{Conséquences pratiques et conclusion}

Malgré la diversité des races utilisées, nous n'avons pas pu mettre en évidence des différences de sensibilité à l'urolithiase d'une race à l'autre, puisque les différents cas sont apparus au hasard dans les deux lots témoins au cours de l'expérience I.

Nos résultats ne nous ont pas permis de confirmer un effet calculigène du sorgho plus intense que celui du maîs; dans l'expérience I le nombre d'agneaux atteints d'urolithiase n'est pas significativement différent dans les deux lots témoins recevant du sorgho ou du mais.

Dans cette expérience, 1'EHDP comme le $\mathrm{NH}_{4} \mathrm{Cl}$ semblent avoir un effet préventif très efficace. Dans l'expérience II où nous n'avons observé aucun cas clinique d'urolithiase, et où aucun calcul n'a pu être décelé à l'abattage, le $\mathrm{NH}_{4} \mathrm{Cl}$, comme 1'EHDP, améliore significativement la croissance des agneaux et réduit leur indice de consommation. On peut alors penser que les agneaux ne recevant aucun traitement sont atteints d'urolithiase à un niveau sub-clinique quii, sans entraîner la formation de calculs visibles lors de l'abattage, a cependant modifié significativement leur vitesse de croissance. La diminution de la teneur en calcium et en phosphore des reins des animaux traités semble confirmer cette hypothèse (tab1. 4), tout comme la corrélation négative existant entre la teneur du rein en calcium et la croissance moyenne journalière chez les animaux du lot $\mathrm{E}$ (fig. I).

A la suite de ces résultats on peut songer à la prévention de l'urolithiase ovine par l'EHDP ou le $\mathrm{NH}_{4} \mathrm{Cl}$. L'EHDP, poudre blanche soluble dans l'eau, serait facile à mélanger aux concentrés distribués aux agneaux. Cependant, son utilisation chez l'animal d'élevage ou de boucherie reste soumise à une réglementation qui n'est pas encore établie. Par contre, dès à présent, on peut souhaiter l'incorporation systématique de $\mathrm{NH}_{4} \mathrm{Cl}$ aux concentrés pour agneaux (incorporation à la dose de I p. roo du poids sec). Ce sel possède en effet un action favorable sur la croissance et l'indice de consommation, même en l'absence de cas cliniques. 


\title{
REMERCIEMENTS
}

Nous remercions le docteur I. Y. Rosenblum (Laboratoires Procter and Gamble, Cincinnati, U. S. A.) qui nous a fourni gracieusement l'EHDP utilisé dans cette expérimentation, qui a pu être effectuée grâce à une subvention de l'Institut technique d'Élevage ovin et caprin (Paris.)

\section{SUMMARY}

\section{OVINE UROLITHIASIS :}

INFLUENCE OF ETHANE-I-HYDROXY-I-DIPHOSPHONATE (EHDP)

AND AMMONIUM CHLORIDE ON ZOOTECHNICAL PERFORMANCES

\author{
AND ON CALCIUM AND PHOSPHATE METABOLISM IN FEED-LOT GROWING LAMBS
}

Two successive experiments were performed to study the influence of EHDP and of $\mathrm{NH}_{4} \mathrm{Cl}$, on daily weight gain, feed consumption, and calcium and phosphate metabolism in young male growing lambs, of various breeds, fed with sorghum or indian corn.

In the first experiment $4^{8}$ lambs were used. They were divided in 4 groups (A, B, C, D). The composition of the diet for each group is given in table $\mathrm{r}$. The number of cases of urolithiasis observed in lambs fed with indian corn (6 cases) or sorghum (5 cases) was not statistically different. No renal stone could be detected (even at slaughtering) in lambs fed with sorghum and EHDP (ro mg/kg of body weight/day) or sorghum and $\mathrm{NH}_{4} \mathrm{Cl}$ (I per roo of the dry weight of the concentrate).

An increased phosphaturia seemed to be the most constant metabolic change preceding the appearance of clinical symptoms of urolithiasis. In lambs fed with sorghum and EHDP, phosphaturia and phosphate content of the kidney was significantly reduced, while calciuria was significantly increased, comparatively to values obtained in lambs fed with sorghum alone (tables 3 and 4 ). $\mathrm{NH}_{4} \mathrm{Cl}$ increased significantly calciuria, calcium retention (table 3 ), the volume of urine excreted, while it decreased significantly the urinary $\mathrm{pH}$ (table 7 ).

In the second experiment 3 groups of I 5 lambs were used (table 1 ). Group $E$ was fed with Indian corn (control animals) ; group G received EHDP (ro mg/kg/day); group F received $\mathrm{NH}_{4} \mathrm{Cl}$ (I p. Ioo of the dry weight of the concentrate (table I). No case of urolithiasis was observed, but the mean daily weight gain of the I5 control animals was significantly smaller than in lambs fed with EHDP or $\mathrm{NH}_{4} \mathrm{Cl}$ (table 2). EHDP and $\mathrm{NH}_{4} \mathrm{Cl}$ decreased the calcium and phosphate kidney contents (table 3 ). In control lambs, a highly significant negative correlation existed between the mean daily gain of body weight and the calcium content of the kidney (fig. I). This seemed to indicate that subclinical urolithiasis was present in control lambs, and might be prevented by EHDP or $\mathrm{NH}_{4} \mathrm{Cl}$.

\section{RÉFÉRENCES BIBLIOGRAPHIQUES}

Barlet J. P., 1972. Effect of porcine, salmon and human calcitonin on urinary excretion of some electrolytes in sheep. J.Endocr., 44, I53-I6r.

Barler J. P., CARE A. D., r972. The influence of parathyroid hormone on urinary excretion of calcium, magnesium and inorganic phosphorus in sheep. Horm. Metab. Res., 4, 315-316.

BerRy J.-P., 1970. Néphrocalcinose expérimentale par injection de parathormone. Étude au microanalyseur à sonde électronique. Néphron, $7,97-$ I 6 .

Braithwaite G. D., I972. The effect of ammonium chloride on calcium metabolism in sheep. $B r . J$. Nutr., 27, 20I-209.

Bulusu L. Hodgkinson A., Nordin B. E. C., Peacock M., I97o. Urinary excretion of calcium and creatinine in relation to age and body weight in normal subjects and patient with renal calculus. Clin. Sci., 38, 601-612. 
Bushman D. H., Emerick R. J., Embry L. B., I965. Experimentally induced ovine phosphatic. urolithiasis : relationships involving dietary calcium, phosphorus and magnesium. $J$. Nutr., 87, 499504 .

Crookshank H. R., I966. Effect of sodium or potassium on ovine urinary calculi. J. Anim. Sci., 25, 1005-1009.

Crookshank H. R., 1970. Effect of ammonium salts on the production of ovine urinary calculi. $J$. Anim. Sci., 30, 1002-1004.

Drach G. W., Boyce W. H., I972. Nephrocalcinosis as a source for renal stone nuclei. Observations on humans and squirrel monkeys and on hyperparathyroidism in the squirrel monkey. J. Urol. 107, 897-904.

Elam C. J., Ham W. E., Schneider B. H., I957. Influence of dietary sodium chloride on incidence of urinary calculi in sheep. Proc. Soc. Exp. Biol. Med., 95, 769-772.

Field A. C., r969. Urinary calculi in ruminants. Proc. Nutr. Soc., 28, I98-202.

Fleisch H.,Bisaz S., CARE A. D., r964. Effect of orthophosphate on urinary pyrophosphate excretion and the prevention of urolithiasis. Lancet, 1, ro65-I067.

Eleisch H., Bonjour J. P., I97I. In KLotz H. P. Les hormones et le calcium, 345³56, Expansion scientifique française, Paris.

Fleisch H., Neuman W. F., r96r. Mechanisms of calcification : role of collagen, polyphosphates, and phosphatases. Amer. J. Physiol., 200, 1296-1 300.

Fleisch H., Russel R. G. G., Bisaz S., Muhlbauer R. C., Williams D. A., i970. The inhibitory effect of phosphonates on the formation of calcium phosphonate crystals in vitro and on aortic and kidney calcification in vivo. Europ. J. Invest., 1, 12-18.

Fraser D., Russel R. G. G., Pohler O., Robertson W. G., Fleisch H., I97I. The influence of disodium ethane-I-hydroxy-I, I-diphosphonate (EHDP) on the development of experimentally induced urinary stones in rats. Clin. Sci., 42, I97-207.

Gasser A. B., Morgan D. B., Fleisch H. A., Richelle L. J., i972. The influence of two diphosphonates on calcium metabolism in the rat. Clin. Sci., 48, 31-45.

Goulding A., Malthus R. S., I969. Effect of dietary magnesium on the development of nephrocalcinosis in rats. $J$. Nutr., 87, 353-358.

Goulding G., Malthus R. S., 1970. Effects of urinary acidity on the development of nephrocalcinosis in rats. Aust. J. Exp. Biol. Med. Sci., 48, 30I-3II.

Gyory A. Z., Edwardes K. D. G., Robinson J., Palmer A. A., I97o. The relative importance of urinary $\mathrm{pH}$ and urinary content of citrate magnesium and calcium in the production of nephrocalcinosis by diet and acetazolamide in the rat. Clin. Sci., 39, 605-623.

HoAR D. W., EMERcix R. J., EMBRY L. B., r97o. Influence of calcium source, phosphorus level and acid base forming effects of the diet on feedlot performance and urinary calculi formation in lambs. J. Animal Sci., 31, I18-125.

Jean-Blain M., Jean-Blain A., Jean-Blain C., r968. La sédimentose, un aspect de la lithiase urinaire des animaux de l'espece ovine. Revue Méd. vét., 31, 623-65I.

Jung A., Russell R. G. G., Bisaz M., Morgan D. B.,(Fleisch H., r97o. Fate of intravenously injected pyrophosphate ${ }^{38-} \mathrm{P}$ in dogs. Amer. J. Physiol., 218, 1757-1764.

KING W. R., Francis M. D., Michael W. R., r97I. Effect of disodium ethane-r-hydroxy-I, I-diphosphonate on bone formation. Clin. Orthop. Rel, Res., 78, 25 I-270.

Lavan J. M., 1969. The effect of oral ammonium chloride on the urinary excretion of calcium, magnesium and sodium in man. Irish J. Med. Sci., 2, $223-227$.

Manston R., I97r. Body fluid volumes in goats and the effect of oral doses of ammonium chloride. Res. Vet. Sci., 12, I68-I69.

Michael W. R., KIng W. R., Wakim J. R., I972. Metabolism of disodium-ethane-I-hydroxy-I, I-diphosphonate (disodium etidronate) in the rat, rabbit, dog and monkey. Clin. Orthop. Rel. Res. (sous presse).

Packet L. V., Butcher G. A., r963. Effects of dietary sodium citrate and oxytetracycline upon fattening lambs. J. Anim. Sci, 22, r roo-1105.

Parfit A. M., 1969. Acetazolamide and sodium bicarbonate induced nephrocalcinosis and nephrolithiasis. Ann. Intern. Med, 124, 736-740.

Robisins J. D., KUnkel H. O., CRookshank H. R., 1965. Relationships of dietary mineral intake to urinary mineral excretion and the incidence of urinary calculi in lambs. $J$. Anim. Sci., 24, 76-82.

Schibler D.,Russel R. G. G., Fleisch H., I968. Inhibition by pyrophosphate and polyphosphatic of aortic calcification induced by vitamin $\mathrm{D}_{3}$ in rats. Clin. Sci., 35, 363-372.

Udall H. R., Chen Chow F. H., I965. Studies on urolithiasis. VII. The effects of sodium, potassium or chloride ions in control of urinary calculi. Cornell Vet., 55, 538-544.

Udall R. H., Chen Chow F. H., 1969. The etiology and control of urolithiasis. Adv. Vet. Se

Comp. Med., 18, 29-56. 